\title{
De las Guerras a los Conflictos Armados Internos: la Guerra del Pacífico y el Conflicto Armado Interno Colombiano
}

\author{
From the Wars to the Internal Armed Conflicts: the Pacific War and the Colombian Internal \\ Armed Conflict \\ ${ }^{a}$ Andrés Felipe Rivera Ramírez ${ }^{73}$ \\ a ariverar@unicartagena.edu.co “Semillero Políticas Públicas Participación y Desarrollo, Programa de Derecho, Universidad de Cartagena. \\ Cartagena, Colombia.
}

Forma de Citar: A.F. Rivera-Ramírez, "De las Guerras a los Conflictos Armados Internos: la Guerra del Pacífico y el Conflicto Armado Interno Colombiano", Rev. Saberes, Vol. 13, No. 02, pp. 106 - 114, 2020.

Recibido: 24/03/2020 Evaluación: 28/05/2020 Aceptado:30/06/2020 DOI: https://doi.org/10.25213/1794-4384/1302.015

\section{Resumen}

Un conflicto armado internacional implica el enfrentamiento entre dos o más estados, los cuales quieren imponer su voluntad a través del uso de la fuerza. La guerra del Pacífico fue una lucha entre tres (3) naciones hermanas, Chile, Perú y Bolivia. Las causas se pueden delimitar en tres (3): Incumplimiento de tratados internacionales anteriormente ratificados, interés por territorios fronterizos y descontento por los limites heredados por parte de la corona española en la época colonial. La pugna tuvo lugar tanto en escenarios terrestres como marítimos y produjo las ya conocidas consecuencias de cualquier enfrentamiento bélico. Sin embargo, algunas partes salieron mucho más beneficiadas al final de la guerra. Igual hecho sucedido en el conflicto interno colombiano, el cual tuvo una trascendencia histórica y una serie de consecuencias paralelas con el conflicto internacional anteriormente mencionado. Este trabajo busca analizar el hito histórico ocurrido.

\section{Palabras Clave}

Guerra, conflicto, tratado, territorio, acuerdos internacionales.

\begin{abstract}
An armed conflict implies the confrontation between two or more States, which want to impose their will through the use of force. The Pacific War was a struggle between three sister nations, namely: Chile against Peru and Bolivia. The causes can be delimited in three: Non-compliance with previously ratified international treaties, interest in border territories and dissatisfaction with the limits inherited by the Spanish crown in the colonial era.

The struggle took place both in land and sea scenarios and produced the well-known consequences of any military confrontation, however, some parts came out much more benefited at the end of the war.
\end{abstract}

\section{Keywords}

War, conflict, treaty, campaigns, international agreements.

\section{Introducción}

La comunidad latina está estrechamente relacionada. Desde los inicios de la vida colonial, los estados integrantes del cono sur de América han venido trazando sus fronteras con

\footnotetext{
${ }^{73}$ Autor para correspondencia: correo electrónico ariverar@unicartagena.edu.co 
influencia de la corona española y portuguesa, respectivamente. Al inicio de la vida republicana de las distintas naciones recientemente independizadas de los imperios europeos, comenzaron las primeras inconformidades respecto a la distribución territorial. Algunos estados reclamaban territorios que ancestralmente les pertenecían antes de la ocupación colonial. Sin embargo, algunos otros argumentaban que ya se habían arraigado y establecido en dichos asentamientos y por tanto les pertenecían. En esta pugna entran los estados chileno y peruano, desarrollando un conflicto bélico y diplomático que generaría una serie de consecuencias que ocurrió y que cuando se analiza y se compara con otros tipos de conflicto como por ejemplo, el conflicto interno colombiano el cual presenta lugares comunes.

Así pues, estudiar los precedentes, las causas, el desarrollo de la guerra, las consecuencias, entre otros, con el objetivo de analizar y hacer el paralelo con el conflicto armado interno colombiano en el marco de los acuerdos firmados en el 2016. Para tales efectos hemos dividido la ponencia en los siguientes apartes I. Chile vs Perú; La guerra del Pacifico. II. Desarrollo de la guerra. III. Consecuencias. IV. Conclusiones. V. Anexos. VI. Glosario. VII. Bibliografía.

\section{Chile vs Perú: la Guerra del Pacífico}

Para comenzar con el desarrollo de la temática, se hace necesario delimitar los siguientes términos definidos oficialmente en los convenios de Ginebra:

- El conflicto armado interno comprendería las acciones armadas en el interior de un estado que dan lugar a hostilidades dirigidas contra un gobierno legal, que presentan un carácter colectivo y un mínimo de organización.

- El conflicto armado internacional es aquel en que se enfrentan "Altas Partes Contratantes", en el sentido de estados. Un Conflicto armado internacional (en adelante CAI) ocurre cuando

\footnotetext{
${ }^{74}$ Aldazosa, A. V. A. V. A. e. P. d. I. B. (2016). LA Referencia. [Data base]. EL IMPUESTO AL SALITRE Y LA GUERRA DEL PACÍFICO.
}

uno o más estados recurren a la fuerza armada contra otro, sin tener en cuenta las razones o la intensidad del enfrentamiento.

La Guerra del Pacifico fue un conflicto armado internacional ocurrido durante los años 1879 y 1883 en el que Chile se enfrentó contra Bolivia y Perú. Fue llamada guerra del Salitre y el Guano debido a la disputa de esta materia prima por parte de los estados en cuestión. Esta guerra se llevó a cabo en el océano Pacífico, el desierto de Atacama en Chile y en los valles y serranías de Perú.

En territorio boliviano se encontraban empresas chilenas y británicas que explotaban el mineral del salitre y el guano (Se utiliza principalmente en la fabricación de Ácidos Nítrico y Sulfúrico y Nitrato de Potasio. Además, es un agente oxidante y se usa en agricultura como fertilizante nitrogenado que puede reemplazar a la urea por su alto contenido en Nitrógeno). La guerra estalló cuando ante la negativa de Bolivia de revocar la disposición de nuevos impuestos, decide rematar los bienes chilenos retenidos por lo que en consecuencia, hubo una reacción militar por parte de Chile. A todo esto, Perú había firmado un tratado de alianza defensiva con Bolivia, lo que lo llevó a ponerse en contra de Chile.

Luego de varias batallas y combates, entre 1883 y 1884, Chile, Perú y Bolivia acceden a firmar un pacto de tregua que puso fin a la guerra luego de cuatro (4) años. Los 3 países debieron acceder a nuevas delimitaciones territoriales y tratados políticos que se acordaron entre todas las partes. Quién más salió beneficiado fue Chile, que logró conquistar diversos territorios y obtener ventajas económicas. ${ }^{74}$

\section{- Causas de la Guerra del Pacifico}

Una serie de sucesos llevaron a que se desencadenara esta guerra, entre ellos están los siguientes:

- Delimitación fronteriza defectuosa que venía de tiempos coloniales entre Bolivia y Chile.

http://lareferencia.info/vufind/Record/BR def4ab1a23572c7f75 25e37beb4bdb34. 27.12.2019. 6:36 p.m.

Página | 108 
- La explotación de las riquezas por parte de capitales chilenos en el territorio donde no estaban bien precisados los límites.

- Bolivia y Perú firmaron un pacto de alianza en 1873, donde se garantizaban la integridad de los territorios y el apoyo ante amenazas militares.

- Los acuerdos del tratado chileno-boliviano del año 1874 que fueron incumplidos por parte de Bolivia.

- Tanto Chile como Bolivia querían apoderarse de los yacimientos de salitre existentes en la zona.

- La confiscación de los bienes pertenecientes a las compañías mineras de Chile y el remate de las salitreras, el cual fue ordenado por el general y presidente de Bolivia Hilarión Daza.

\section{- Desarrollo de la guerra}

Campaña marítima (1879): la Guerra del Pacífico se desarrolló bajo distintos escenarios. El de más importancia fue el de mar, en el Océano Pacífico. Los chilenos tenían claro que tener el dominio del mar era una clara ventaja que tenían en aquel conflicto armado. Por esa razón, se lanzaron en la conquista del Océano pacífico. Se iniciaron las campañas marítimas, en la que comenzaron a bloquear el Puente de Iquique, principal puerto salitrero de Perú. La campaña marítima del año 1879 representó en dos combates: el combate Naval de Iquique y el combate de Angamos.

Campaña de Tarapacá (1879): el 2 de noviembre del año 1879 el general Erasmo Escala y el ministro de guerra Rafael Sotomayor, desembarcaron en la Pisagua con más de 10.000 hombres y 16 buques transportados de guerras. De esta manera inició el avance de las tropas de Chile en el territorio enemigo. Se llevaron a cabo dos enfrentamientos, la Batalla de Dolores y el combate de Tarapacá. En el primero, Sotomayor quedó con 187 heridos y 62

\footnotetext{
75 Enciclopedia de historia. (2015). Guerra del pacifico. Recuperado de https://enciclopediadehistoria.com/guerra-delpacifico/. 04.01.2020. 9:20 p.m.
}

muertos. En el combate de Tarapacá las fuerzas chilenas sufrieron una derrota, pero no significó un retroceso para el pueblo austral.

Campaña de Tacna y Arica (1880): en el año 1880 se llevó a cabo la campaña de Tacna y Arica, la cual resultó ser un acontecimiento determinante en la victoria de los chilenos. Además, esta campaña marcó la desunión de Perú y de Bolivia.

Batalla de Tacna: Ocurrida el 26 de mayo, diez mil chilenos atacaron a los peruanos y bolivianos, estaban al mando de Baquedano. La victoria fue inminente. Mientras los peruanos se retiraban a Arequipa, los bolivianos se retiraban por completo para no participar más en esta guerra.

Toma de Arica: Para conseguir la verdadera victoria, la idea era tomar Arica y ocupar la fortificada plaza de la ciudad. El 7 de junio llevaron a cabo este plan, una de las acciones más violentas de la campaña de la Guerra del Pacífico. El 22 de octubre el gobierno de Estados Unidos intervino, convocando una conferencia de paz, en la que ninguno de los tres países estuvo dispuesto a ceder.

La Campaña de Lima (1880): los primeros días de noviembre José Francisco Vergara encargó que se prepararan las fuerzas de Chile para que entraran en la capital peruana. El 20 de noviembre desembarcaron en Paracas, localidad que está a $200 \mathrm{~km}$ de Lima, desde allí comenzaron a avanzar a los pueblos sin mostrar tanta oposición. El 13 de enero de 1881 se enfrentaron a las fuerzas peruanas en las siguientes batallas: ${ }^{75}$ 1) La batalla de San Juan, 2) Asalto de Morro Solar, 3) Batalla de Chorrillos.

Batalla de Miraflores (1881): Chile ya había perdido a muchos guerreros y Baquedano no quería seguir lo que envió a Isidoro Errázuriz para ofrecerle la paz a Piérola. Sin embargo, esa ofrenda de paz no resultó, Perú ya estaba preparado para atacar al pueblo chileno. Esa batalla fue desigual, Chile no contaba con muchos hombres. Pero, llegaron refuerzos y 
lograron dar un duro golpe a las tropas peruanas.

Campaña de la Sierra (1881-1884): último combate de la Guerra del Pacífico y la más larga. Se llevó a cabo en las Sierras peruanas, comenzó en abril del 1881 hasta junio del 1884.

Combate de la Concepción: es el combate más dramático de esta Guerra del Pacífico, se efectuó el 9 y 10 de julio del 1882 en el pueblo La Concepción, Perú. El pueblo de Chiles resistió por dos días el ataque de los soldados del bando de los peruanos. Aunque murieron muchos chilenos, al final, chile derrotó de manera definitiva en la Batalla de Huamachuco. Luego de esto, se firmó el tratado Ancón en el 1883 para poner final a la Guerra con Perú. ${ }^{76} 77$

\section{- Consecuencias de la Guerra del Pacífico}

Esta guerra dejó muchas consecuencias territoriales, sociales y económicas entre los países beligerantes, las cuales se consideran a continuación:

\section{- Consecuencias Territoriales}

Entre las principales consecuencias territoriales se encuentran:

- Chile extendió su territorio al quedarse con Tarapacá y Arica.

- Luego de 1925, Perú logra recuperar el territorio de Tacna, que hasta entonces estaba en manos de Chile por la guerra. ${ }^{78}$

- La provincia de Tarata fue devuelta a Perú en 1925 como consecuencia de la guerra.

- Se fijaron los límites entre Bolivia y Chile, en donde Bolivia acepta la anexión de chile en la franja boliviana.

- Bolivia pierde su única salida al mar.

- Chile y Argentina se reparten la Puna de Atacama que fue cedida por Bolivia.

\section{- Consecuencias sociales}

La guerra del Pacífico entre Bolivia, Chile y Perú trajo las siguientes consecuencias sociales:

\footnotetext{
${ }^{76}$ Historia Peruana. (2018). Guerra con Chile o del Pacífico. Recuperado de: https:/historiaperuana.pe/periodoindependiente/republica/guerra-peru-chile-del-pacifico/amp/. 15. 01. 2020. 9:15 p.m.

${ }^{77}$ Ver anexos. Mapa uno (1).
}

- La guerra del Pacífico trajo alrededor de 15.000 muertos entre civiles y militares.

- Se comenzó a incorporar a la comunidad indígena a la sociedad peruana.

- Se desencadenó una gran guerra Civil en Perú debido a las diferencias sociales entre los diferentes sectores de la población surgida a partir de esta guerra.

\section{- Consecuencias Económicas}

- Bolivia se vio obligada a devolver los bienes embargados a Chile, que causaron el inicio de la guerra.

- Chile se vio beneficiada económicamente al poder controlar territorios con acceso del salitre $\mathrm{y}$ diversos minerales. Además de poder controlar zonas marítimas y sus aranceles.

- Durante la guerra, Chile logró bloquear toda la zona dónde se producía salitre, guano y azúcar, lo que dejó en la ruina económica a Perú, que se abastecía de dicha zona.

- Bolivia se vio debilitado económicamente por la pérdida de la salida al mar y por la pérdida de territorios.

\section{- Consecuencia Histórica}

Este conflicto fue el inicio de una larga disputa judicial en instancias internacionales por parte de los estados beligerantes. No obstante, chilenos y peruanos fueron quienes recurrieron a la Corte internacional de Justicia de la Haya. El fallo de la Corte de La Haya sobre la delimitación marítima entre Chile y Perú es una sentencia dictada por la Corte Internacional de Justicia el 27 de enero de 2014, mediante la cual se resolvió el caso concerniente a la delimitación marítima entre la República del Perú y la República de Chile, iniciado por la primera el 16 de enero de 2008, que involucraba un área marítima y su correspondiente espacio aéreo de aproximadamente $67139,4 \mathrm{~km}^{2}$, de los cuales

\footnotetext{
${ }^{78}$ The Bullonist. (2000). Wikisourse. [Data base]. Sobre la guerra del Pacífico. Recuperado de: https://es.wikisource.org/wiki/Editorial del diario \%22The Bul lonist $\% 22$ sobre la guerra del Pac\%C3\%ADfico. 28.11.2019. 5:07 p.m.
} 
unos $38000 \mathrm{~km}^{2}$ eran considerados como mar chileno y $28471,86 \mathrm{~km}^{2}$ como alta mar. ${ }^{79}$

Por la sentencia se decidió que el punto de inicio del límite marítimo entre el Perú y Chile es la intersección del paralelo geográfico que cruza el Hito n. ${ }^{\circ} 1$ con la línea de bajamar, y que la frontera marítima sigue el paralelo que pasa sobre el Hito n. $^{\circ} 1$ hasta un punto situado 80 millas marinas de distancia, a criterio de la corte dicha decisión se encuentra sustentada en los acuerdos jurídicamente vinculantes y la práctica bilateral entre ambos países que prueban la existencia de una delimitación marítima efectuada por las partes. Después de la milla 80 la corte fija de nuevo un límite que continúa en dirección suroeste sobre una línea equidistante desde las costas de ambos países hasta su intersección con el límite de las 200 millas marinas medidas desde las líneas de base de Chile y posteriormente, continúa hacia el sur hasta el punto de intersección con el límite de las 200 millas marinas medidas desde las líneas de base de ambos países. La Corte emite su sentencia sin determinar las coordenadas geográficas precisas, disponiendo que sean las propias partes las que procedan a determinar tales coordenadas de conformidad con el fallo, lo cual ocurrió el 25 de marzo de 2014.

En consecuencia, adjudicó al Perú un área marítima total de algo más de $50000 \mathrm{~km}^{2}$, que pasó a formar parte de las zonas marítimas peruanas, y que hasta entonces se distribuía en dos sectores de condición jurídica distinta: el primer sector, con una superficie aproximada de $22000 \mathrm{~km}^{2} 56$, era considerado por Chile como parte de su zona económica exclusiva, y por tanto, explotado como mar patrimonial; en cambio, el segundo sector, de $28471,86 \mathrm{~km}^{2} 5$, era considerado por Chile como parte de la alta mar, es decir, un área oceánica abierta a todos los países. Como contraparte, el fallo ratificó la soberanía y los derechos soberanos de Chile sobre $16352 \mathrm{~km}^{2}$ de mar que ya poseía y explotaba desde hacía décadas, siendo esta mayormente el área situada a menor distancia a la costa y que, por tanto, corresponde a la más

\footnotetext{
79 Dipublico.org (2015). Disputa Marítima (Perú v. Chile). Fallo de la Corte Internacional de Justicia (en español). Recuperado de: https://www.dipublico.org/102630/disputa-maritima-peru-vchile-fallo-de-la-corte-internacional-de-justicia-enespanol/27.01.2020. 6:00 p.m.
}

rica en recursos pesqueros del total de aguas en controversia. ${ }^{80}$

$[\text { ver mapa anexo] }]^{81}$

\section{Relación con el conflicto armado colombiano}

Los conflictos armados, tanto internos como internacionales, guardan una estrecha relación en todos los aspectos de una guerra. Sin embargo, la guerra chileno peruana y la interna colombiana, coinciden mayoritariamente en las consecuencias y la terminación.

Podemos hacer un paralelo entre las consecuencias que dejó para los Estados en conflictos en la guerra del pacifico y lo que nos dejó cincuenta años de conflicto armado interno con la guerrilla de las Farc.

\section{- Consecuencias Territoriales}

La composición territorial de Chile, Perú y Bolivia se modificó a raíz de la guerra. Chile obtuvo tierras que anteriormente les pertenecían a los otros Estados. Por su parte, Perú y Bolivia sufrieron importantes pérdidas. Empero, este punto no es análogo entre ambos conflictos, ya que, si bien, el territorio colombiano no mutó en la división política, fueron factores como la minería ilegal y los cultivos ilícitos los que afectaron el medio ambiente y volvieron a zonas habitadas en áreas desocupadas por la población civil debido a los desplazamientos. Tal fue el caso de municipio de Barrancabermeja en Santander, en la región del Magdalena Medio. Una característica de la dinámica territorial del conflicto armado en este periodo es que la frontera con Venezuela, desde el municipio de Puerto Carreño en el Vichada, pasando por la región del Catatumbo, la serranía del Perijá, hasta el municipio de Riohacha en La Guajira; ya se ve significativamente afectada por la intensidad del conflicto armado, bosquejando así un corredor fronterizo de disputa territorial, que más adelante se definirá

\footnotetext{
80 BBC News. (2014). ¿Qué ganaron y perdieron Perú y Chile con el fallo de La Haya? Recuperado de: https://www.bbc.com/mundo/noticias/2014/01/140127_chile_pe ru_cij_fallo haya_az/. 25.01.2010.6:14 p.m. ${ }^{81}$ Ver anexos. Mapa tres (3).
}

Página | 111 
con más claridad. ${ }^{82} \mathrm{El}$ acuerdo de la Habana, en los principios, buscaba la democratización de la tierra y el retorno de sus propietarios iniciales

\section{- Consecuencias Sociales}

La Guerra del Pacifico dejó miles de víctimas, entre los combatientes y la población civil. Victimas las cuales no tuvieron una efectiva reparación, en ninguno de los Estados beligerantes. El conflicto interno, debido a su larga duración, multiplicó con creces las cifras de víctimas manejadas en la guerra internacional. El Centro Nacional de Memoria Histórica (CNMH) da cuenta de 352.786 hechos documentos en medio del conflicto armado, entre 1958 y 2018. El total de víctimas fatales fueron 261.619 y están divididos así: 214.584 civiles, 46.675 combatientes y 360 personas sin información. ${ }^{83}$ En materia de víctimas mortales, el acuerdo de la Habana plantea en su página 135, la amnistía y el indulto para las muertes en combate en el marco del DIH. Sin embargo, las muertes que se salgan de este serán materia de estudio de otras jurisdicciones.

\section{- Consecuencias Internacionales}

Al igual que en el conflicto chileno-peruano, el conflicto armado interno trajo una serie de consecuencias a escala internacional para el Estado colombiano, entre estas están sentencias de tribunales internacionales como lo fue la de la Corte Interamericana de derechos humanos caso Rodríguez Vera y otros (desaparecidos del palacio de justicia) vs. Colombia. Esta providencia se refiere a las presuntas desapariciones forzadas de 12 personas ocurridas durante la toma y retoma del Palacio de Justicia ocurridas el 6 y 7 de noviembre de 1985 en Bogotá. Asimismo, sobre la presunta desaparición forzada y posterior ejecución de un Magistrado y sobre la presunta detención y tortura de 4 personas. La Corte declara la

\footnotetext{
82 Salas-Salazar, L. (2016). Conflicto armado y configuración territorial. Bitácora Universidad Nacional, 52(1), 13. Recuperado de http://www.scielo.org.co/pdf/biut/v26n2/v26n2a05.pdf. 5. 02. 2020. 9:30 a.m.

${ }^{83} \mathrm{El}$ Tiempo (22 de octubre de 2018). Cifras del conflicto armado en Colombia en los últimos 60 años. El Tiempo. Recuperado de https://www.eltiempo.com/justicia/investigacion/cifras-del-
}

responsabilidad internacional de Colombia. Los hechos del presente caso se enmarcan en los sucesos conocidos como la toma y la retoma del Palacio de Justicia, en la ciudad de Bogotá, ocurridas los días 6 y 7 de noviembre de 1985. En particular, el caso se relaciona con la presunta desaparición forzada de Carlos Augusto Rodríguez Vera, Cristina del Pilar Guarín Cortés, David Suspes Celis, Bernardo Beltrán Hernández, Héctor Jaime Beltrán Fuentes, Gloria Stella Lizarazo, Luz Mary Portela León, Norma Constanza Esguerra Forero, Lucy Amparo Oviedo Bonilla, Gloria Anzola de Lanao, Ana Rosa Castiblanco Torres e Irma Franco Pineda durante el operativo de retoma. Asimismo, el caso se relaciona con la presunta desaparición y posterior ejecución del Magistrado Carlos Horacio Urán Rojas, así como sobre la presunta detención y tortura de Yolanda Santo domingo Albericci, Eduardo Matson Ospino, Orlando Quijano y José Vicente Rubiano Galvis. Adicionalmente, el caso versa sobre la alegada falta de esclarecimiento judicial de los hechos y la sanción de la totalidad de los responsables. La Corte decide aceptar el reconocimiento parcial de responsabilidad internacional efectuado por el Estado, es responsable por la desaparición forzada, por la violación del deber de garantizar el derecho a la vida, por ejecución extrajudicial, por la violación del derecho a la libertad personal, por la violación de las garantías judiciales y a la protección judicial, entre otros.

\section{Conclusiones}

- La Guerra del Pacifico fue un conflicto de escalas internacionales que marcó un precedente en la vida diplomática y jurídica a nivel internacional en la esfera sur de América. - Bajo este orden de ideas, es de vital importancia que el conflicto armado colombiano sea interpretado y regulado a partir de un enfoque diferencial con respecto a la

\footnotetext{
conflicto-armado-en-colombia-en-los-ultimos-60-anos-283920. 2. $02.2020 .11: 35$ p.m

${ }^{84}$ Gobierno Nacional de Colombia. (2016). Acuerdo final para la terminación del conflicto y la construcción de una paz estable y duradera. Recuperado de

https://www.mesadeconversaciones.com.co/sites/default/files/24 08 2016acuerdofinalfinalfinal-1472094587.pdf
} 
población vulnerable (Ayala, E. T., y Osorio, E. G. ,2016)).

- Las consecuencias de este conflicto han trascendido tanto que aún permanecen disputas judiciales a en los tribunales internacionales entre las naciones en cuestión.

- Como pudimos observar, los conflictos mencionados se encuentran estrechamente relaciones en muchos de sus aspectos principales. El precedente histórico internacional que nos dejó la guerra del pacifico puede ser utilizado como referencia para seguir analizando otros vértices del conflicto armado interno y seguir en la labor académica de cercar todo lo relacionado con la guerra para las futuras generaciones

\section{Referencias Bibliográficas}

Ayala, E. T., y Osorio, E. G. (2016). La mujer como víctima y actor del conflicto armado en Colombia. Perspectivas. 1(1). 73-80.

Aldazosa, A. V. A. V. A. e. P. d. I. B. (2016). LA Referencia. [Data base]. EL IMPUESTO AL SALITRE Y LA GUERRA DEL PACÍFICO. Recuperado de: http://lareferencia.info/vufind/Record/BR_def4 ab1a23572c7f7525e37beb4bdb34

BBC News. (2014). ¿Qué ganaron y perdieron Perú y Chile con el fallo de La Haya? Recuperado de: https://www.bbc.com/mundo/noticias/2014/01/ 140127 chile_peru_cij_fallo_haya_az/

Dipublico.org (2015). Disputa Marítima (Perú v. Chile). Fallo de la Corte Internacional de Justicia (en español). Recuperado de: https://www.dipublico.org/102630/disputamaritima-peru-v-chile-fallo-de-la-corteinternacional-de-justicia-en-espanol/

El Tiempo (22 de octubre de 2018). Cifras del conflicto armado en Colombia en los últimos 60 años. El Tiempo. Recuperado de https://www.eltiempo.com/justicia/investigacio n/cifras-del-conflicto-armado-en-colombia-enlos-ultimos-60-anos-283920

Enciclopedia de historia. (2015). Guerra del pacifico.

Recuperado https://enciclopediadehistoria.com/guerra-delpacifico/

Gobierno Nacional de Colombia. (2016). Acuerdo final para la terminación del conflicto y la construcción de una paz estable y duradera. Recuperado de https://www.mesadeconversaciones.com.co/sit es/default/files/24 08 2016acuerdofinalfinalfi nal-1472094587.pdf

Historia Peruana. (2018). Guerra con Chile o del Pacífico. Recuperado de: https://historiaperuana.pe/periodoindependiente/republica/guerra-peru-chile-delpacifico/amp/

Mapa. Ciudades perdidas. Recuperado de https://chrismielost.blogspot.com/2016/01/ciud ades-perdidas-humberstone-y-el-oro 5.html

Salas-Salazar, L. (2016). Conflicto armado y configuración territorial. Bitácora Universidad Nacional, 52(1), 13. Recuperado de http://www.scielo.org.co/pdf/biut/v26n2/v26n2 a05.pdf

The Bullonist. (2000). Wikisourse. [Data base]. Sobre la guerra del Pacífico. Recuperado de:https://es.wikisource.org/wiki/Editorial_del diario \%22The Bullonist $\% 22$ sobre la_guer ra_del_Pac\%C3\%ADfico.

Video. Pillco, H. [HugoX ChugoX]. (2017, septiembre 17). LA GUERRA DEL PACÍFICO EXPLICADA | antecedentes, causas y consecuencias | [Archivo de video]. Recuperado de https://www.youtube.com/watch?v=99COGFr 7oz0.

\section{Anexos}

1. Mapa. Ciudades perdidas. Recuperado de

https://chrismielost.blogspot.com/2016/01/ciud ades-perdidas-humberstone-y-el-oro $5 . \mathrm{html}$. 26. 01.2020. 7:45 p.m.

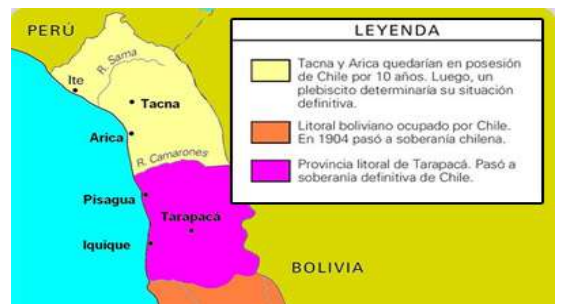


2. Video.Pillco, H. [HugoX ChugoX]. (2017, septiembre 17). LA GUERRA DEL PACÍFICO EXPLICADA | antecedentes, causas y consecuencias | [Archivo de video]. Recuperado de

https://www.youtube.com/watch?v=99COGFr

\section{7oz0. Minuto siete (7:00)}

\section{11. 2019. 3:40 p.m.}

3. Mapa. BBC News. ¿Qué ganaron y perdieron Perú y Chile con el fallo de La Haya? Recuperado

https://www.bbc.com/mundo/noticias/2014/01/ 140127 chile peru cij_fallo haya_az. 26.01.2020. 2:17 p.m.
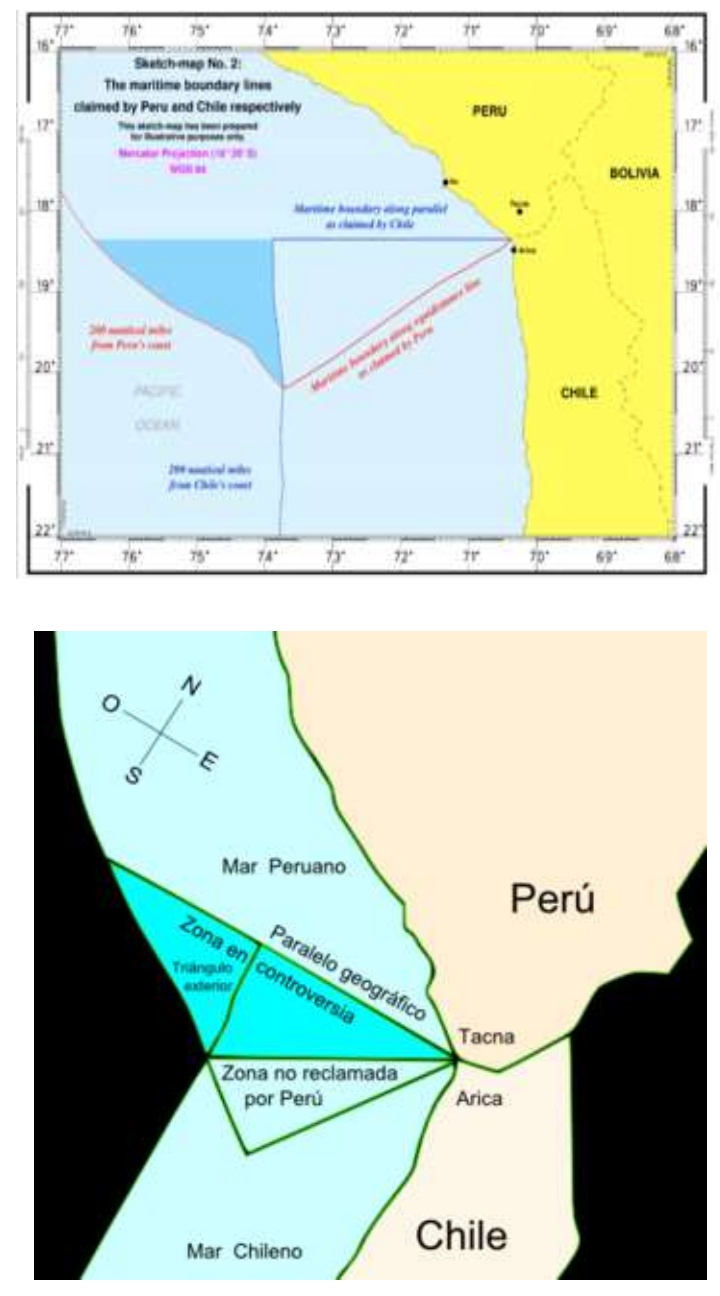